\title{
Thermal properties of high refractive index epoxy resin system
}

\author{
Wei-Fang Su, ${ }^{\text {a,* }}$ Ya-Ching Fu ${ }^{\mathrm{a}}$, Wei-Ping Pan ${ }^{\mathrm{b}}$ \\ ${ }^{\mathrm{a}}$ Department of Materials Science and Engineering, National Taiwan University, Taipei, Taiwan \\ ${ }^{\mathrm{b}}$ Materials Characterization Center, Western Kentucky University, Western Kentucky, KY 42101, USA
}

\begin{abstract}
The goal of our study is to develop high refractive index ( $>1.60)$ epoxy resin system for optoelectronics and electro-optical equipment assemblies. Molecular design approach is applied to reach this goal by implement of rigid rod moeities or heavy elements $(\mathrm{Br})$ into the epoxy structures. The thermal properties of these new epoxy resins were studied by modulated differential scanning calorimetry (MDSC) and thermogravimetric analyzer (TGA) to investigate the effects of changes of chemical structures. The epoxy resin system containing rigid rod structure exhibits better thermal properties than those containing flexible structure. (C) 2002 Elsevier Science B.V. All rights reserved.
\end{abstract}

Keywords: High refractive index; Epoxy resin; Thermal properties

\section{Introduction}

Polymers are now extensively used in optical and electro-optical devices because of their several superior properties over inorganic materials, such as transparency, lightweight, ease of fabrication, and possible molecular tailoring through controlled synthesis. The primary applications of these materials include flat-panel displays, optical fibers, compact/optical disks and so on. These devices generally consist of many optical and electro-optical parts, and the application of adhesives is usually necessary to bond these components.

Adhesives used in the assembly of these optical devices can be divided into two groups [1]. One is those used to join optical paths-sections that pass light and the other is those only used to reinforce the bonding between two components without the passing of light. Adhesives used in the sections with light

\footnotetext{
* Corresponding author.

E-mail address: suwf@ccms.ntu.edu.tw (W.-F. Su).
}

passing are required to minimize the reflection of light. Interference may rise if part of the light beam is reflected at the junction of adhesives, especially when this occurs close to the light source. Thus, in order to minimize the reflection, the requirements of the adhesives that used in the optical circuits not only include the bonding ability but also the optical compatibility with the bonded substrates. It is necessary for them to have high degree of light transmittance and matched refractive indexes as those of the materials they bonded to sustain good optical properties and prevent optical distortion. Thus, in the assembly of optical components, the concern of the refractive indexes of optical adhesives is important in the selection of proper adhesives, they should match those of the substrates to optimize the efficiency of bonding.

Epoxy resins are widely used as adhesives because of their remarkable bonding ability to various materials including metals, glass, ceramics, wood and plastics. The strong bonding forces are attributed to three main properties: (1) strong van der waals forces and 
hydrogen bonding established by the repeating polar sites generated through curing reactions, (2) low chemical condensation shrinkage that minimizes stresses developed at the adhesive interface, and (3) high physical strengths of most epoxy systems.

In addition to the good bonding epoxy can offer, the versatility of modifications is another important advantage for its application as adhesives. Properties of epoxy adhesives such as high temperature strength and stability, peel strength, flame retardance, electrical properties, and even the optical property, refractive index, could be modified by alterations of structure formulation. As optical adhesives, the average refractive indices of conventional epoxy resins are in the range of 1.50-1.56. The goal of this study is to develop epoxy resins with high refractive index for optical equipment assemblies.

The refractive index is a manifestation of an average property of the integral material, which influenced mainly by the molecular weight, density and the inherent molar refractivity of its molecular structure according to the Lorentz-Lorentz equation [2] as shown in the following:

$R=\frac{n^{2}-1}{n^{2}+2} \frac{M}{\rho}$

where $R$ represents the molar refractivity, $n$ the refractive index, $\rho$ the density, and $M$ is the molecular weight. The molar refractivity also related to the polarizability of the molecule.

Many high refractive index polymers have been reported and these systems usually consist of highly conjugated, aromatic rigid rod moiety, $\pi$-electron systems that bear heavy elements such as bromine or iodine [3-7]. Thus in this study, biphenol (BP) epoxy and naphthalene (HP) epoxy were selected because of their rigid rod moiety as well as the heavy element, $\mathrm{Br}$, of tetrabromo bisphenol A epoxy. They are expected to raise the refractive index of epoxy resins.

The selection of an appropriate curing agent is as important as selecting a proper epoxy resin. Curing agents determine the type of chemical bonds formed and the degree of cross-linking within the resin networks. These factors, in turn, affect the chemical resistance, electrical and physical properties of the cured adhesives. Nadic methyl anhydride (NMA) was selected because of its fused rigid ring for high heat resistance and high refractive index. Thermal properties of NMA cured epoxy resin systems were compared with methyl tetrahydrophthalic anhydride (MTHPA) cured systems.

\section{Experimental}

\subsection{Materials}

The structures of the epoxies and curing agents are shown in Fig. 1. HP epoxy and tetrabromo bisphenol A epoxy (BEB350) were supplied by Dainippon Chemicals Inc. and Chang Chun Plastics Co., respectively. 4,4'-Bis(2,3-epoxypropoxy) BP epoxy was synthesized according to literature procedure [8]. Two curing agents, NMA and MTHPA, were supplied by Tokyo Kasei Company.

\subsection{Preparation of samples}

Each set of epoxy systems were melt mixed at equivalent weight with 1 per hundred resin (phr) of imidazole as accelerator. Then they were cured according to their respective curing conditions as shown in Table 1. The curing condition of each sample was determined by differential scanning calorimetry (Dupont Model 910 DSC). The fully cured samples do

Table 1

Cure schedules of epoxy systems

\begin{tabular}{|c|c|c|c|c|c|}
\hline BP/MTHPA & BP/NMA & HP/MTHPA & HP/NMA & BEB/MTHPA & $\mathrm{BEB} / \mathrm{NMA}$ \\
\hline \multicolumn{6}{|c|}{ Curing condition $\left({ }^{\circ} \mathrm{C} / \mathrm{HR}\right)$} \\
\hline $120 / 6$ & $120 / 6$ & $100 / 1$ & $120 / 2$ & $120 / 4$ & $125 / 2$ \\
\hline $160 / 7$ & $160 / 7$ & $120 / 2$ & $160 / 4$ & $160 / 6$ & $160 / 4$ \\
\hline $200 / 4$ & $200 / 4$ & $160 / 4$ & $200 / 4$ & $180 / 2$ & $180 / 6,200 / 4$ \\
\hline
\end{tabular}


4,4'-bis (2,3-epoxypropoxy) biphenyl (BP)

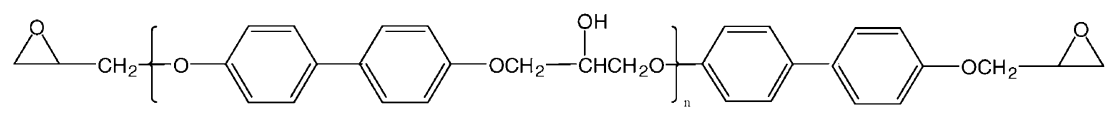

Naphthalene epoxy (HP)

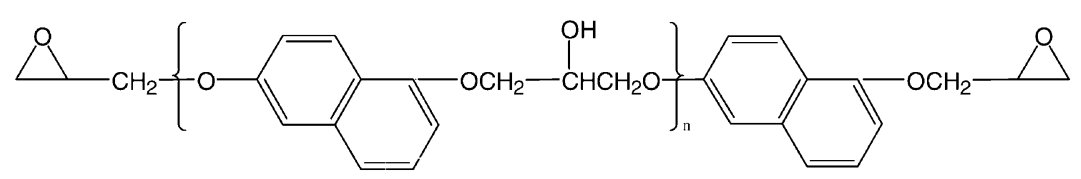

Tetrabrominated Bisphenol A epoxy (BEB-350)

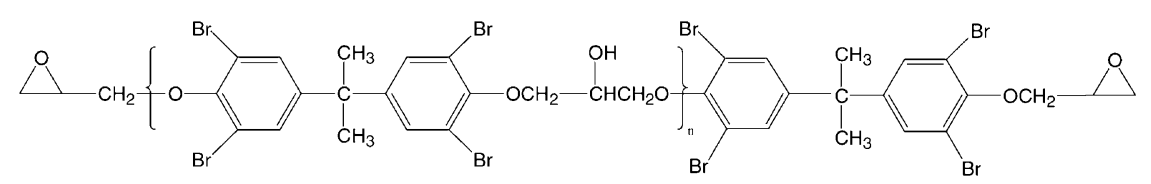

Methyl tetrahydrophthalic anhydride (MTHPA)

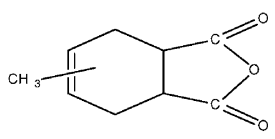

Nadic methyl anhydride (NMA)

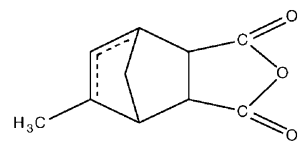

Fig. 1. Structures of epoxies and curing agents.

not exhibit heat of reaction peaks and further increasing in glass transition temperature $\left(T_{\mathrm{g}}\right)$.

\subsection{Instrumentation}

Thermal properties of samples were characterized by modulated differential scanning calorimetry (MDSC) (TA Instrument (MDSC 2910) and thermogravimetric analyzer (TGA) (TA Instrument 2950). Both TGA and MDSC tests were carried out under nitrogen, while TGA ramp $10{ }^{\circ} \mathrm{C} / \mathrm{min}$ from room temperature to $500{ }^{\circ} \mathrm{C}$ and $\mathrm{MDSC}$ ramp $5{ }^{\circ} \mathrm{C} / \mathrm{min}$ from room temperature to the degradation temperatures with modulated heating rate at $\pm 1{ }^{\circ} \mathrm{C} / \mathrm{min}$.

\section{Results and discussion}

Epoxy resins containing bromine atoms are commonly used in electrical laminate, circuit boards, potting, and encapsulation applications because of their good flame retardant property resulted by the bromine atoms. In Fig. 2 and Table 2, tetrabromo epoxy (BEB350) exhibits a higher degradation temperature than BP-type and HP-type epoxy after cured with NMA, though the rate of degradation of BEB350 is higher than that of the others. This might be due to the flexible structure in its backbone, which shows less heat resistant than the rigid backbones, BP and HP group, in the other two epoxy resins. Thus, HP 


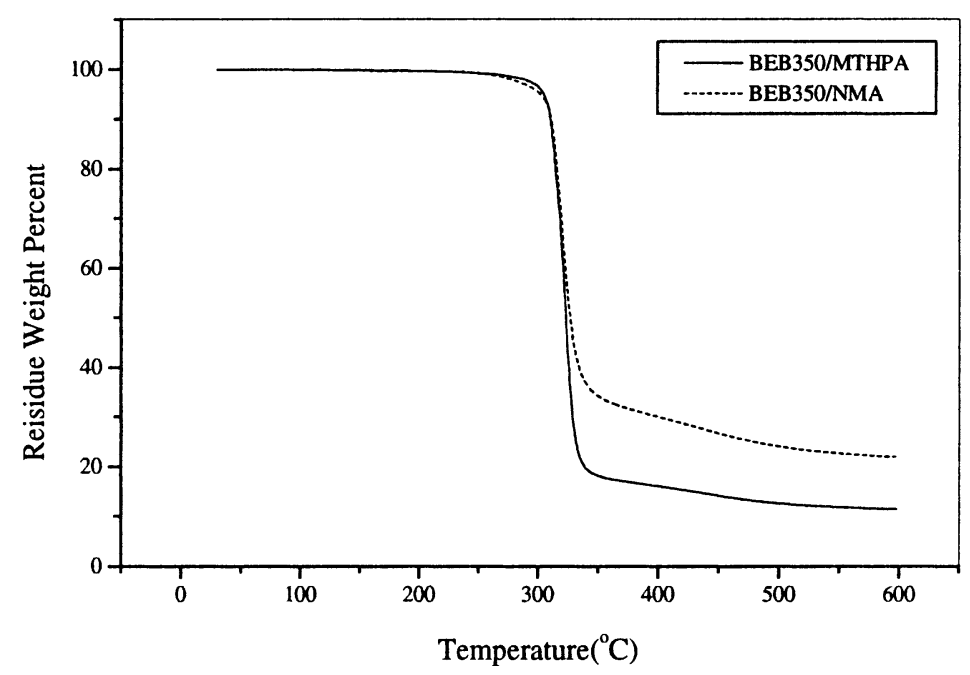

Fig. 2. Results of TGA of various anhydride cured tetrabromo epoxy resins.

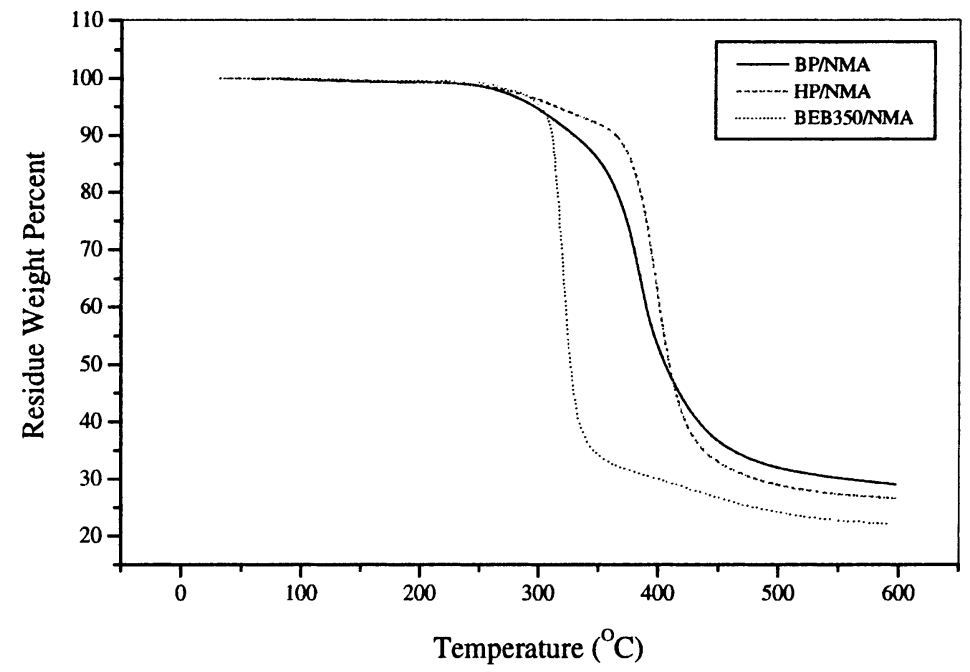

Fig. 3. Results of TGA of NMA cured epoxy resins.

Table 2

Thermal analysis results of MDSC and TGA

\begin{tabular}{llll}
\hline Epoxy system & $T_{\mathrm{g}}\left({ }^{\circ} \mathrm{C}\right)$ & $\begin{array}{l}\text { Loss temperature } \\
\text { at } 5 \text { wt. } \%\left({ }^{\circ} \mathrm{C}\right)\end{array}$ & $\begin{array}{l}\text { Residue wt.\% } \\
\text { at } 600{ }^{\circ} \mathrm{C}\end{array}$ \\
\hline BP/MTHPA & 141 & 307.2 & 15.0 \\
BP/NMA & 162 & 295.7 & 29.1 \\
HP/MTHPA & 145 & 296.0 & 10.5 \\
HP/NMA & 174 & 314.5 & 26.6 \\
BEB350/MTHPA & 160 & 304.0 & 11.4 \\
BEB350/NMA & 184 & 301.4 & 22.0 \\
\hline
\end{tabular}

reserves more residue than $\mathrm{BP}$ and $\mathrm{BEB} 350$ after heating to high temperature, $600{ }^{\circ} \mathrm{C}$. Comparing the thermal stability of epoxy networks cured with two different curing agents, Fig. 3 shows no specific different degradation pattern between HP/NMA and HP/MTHPA. NMA cured network retained more residue at $600{ }^{\circ} \mathrm{C}$ as expected from its rigid structure. Comparison between Figs. 2 and 3 indicates that the degradation pattern was determining by the epoxies rather than by curing agents. 
MDSC results show an increasing trend of $T_{\mathrm{g}}$ from BP to HP, then to BEB350. The BEB350 exhibits the highest $T_{\mathrm{g}}$, this might be due to the steric hindrance effect of $\mathrm{Br}$ atom. Types of curing agents also play important roles in determining the $T_{\mathrm{g}}$. Three NMA cured epoxies averagely show an about $20^{\circ} \mathrm{C}$ higher value of $T_{\mathrm{g}}$ than MTHPA cured resins. This indicates that cross-linking bonds formed by rigid structure in resin network could restrict the molecular motion to have high $T_{\mathrm{g}}$.

\section{Conclusions}

Epoxy resins cured with NMA exhibit about a $20{ }^{\circ} \mathrm{C}$ higher $T_{\mathrm{g}}$ value and retain more residue wt.\% at $600{ }^{\circ} \mathrm{C}$ than those cured with MTHPA. This is due to the more rigid backbone formed by NMA in the resin networks. Among three different types epoxies, the $T_{\mathrm{g}}$ of cured resins tends to increase from BP to HP then to BEB350. The initial weight loss temperature of BEB resins are higher than HP and BP resins due to the lower bond movement of heavy $\mathrm{Br}$ atom. They degrade rapidly after the initial weight loss, while HP and BP exhibit a gradual weight loss over a higher temperature range. This might be due to the rigid main chain structures of HP and BP resins.

\section{Acknowledgements}

Financial supports from National Science Council of Republic of China under Grant NSC89-2218-E002-072 and Western Kentucky University Visiting Scholarship Program are highly appreciated.

\section{References}

[1] N. Murata, in: Proceedings of the Conference on Electronic Components and Technology, 25-28 May 1998, IEEE, pp. $1178-1185$.

[2] J.C. Seferis, in: J. Brandrup, E.H. Immergut (Eds.), Polymer Handbook, 3rd Edition, Wiley, New York, 1980, pp. 451461.

[3] R.A. Gaudiana, R.A.J. Minns, Macromol. Sci. Chem. A28 (9) (1991) 831-842.

[4] R.A. Minns, R.A.J. Gaudiana, Macromol. Sci. Pure Appl. Chem. A29 (1) (1992) 19-30.

[5] B. Wang, A. Gungor, A.B. Brennan, D.E. Rodrigues, J.E. McGrath, G.L. Wilkes, Polym. Prepr. (Am. Chem. Soc. Div. Polym. Chem.) 32 (3) (1991) 521-522.

[6] B. Wang, G.L. Wilkes, J.C. Hedrick, S.C. Liptak, J.E. McGrath, Macromolecules 24 (1991) 3449-3450.

[7] R. Sinta, R.A. Gaudiana, H.G.J. Rogers, Macromol. Sci. Chem. A26 (5) (1989) 773-785.

[8] W.-F. Su, K.C. Chen, S.Y. Tseng, J. Appl. Polym. Sci. 78 (2000) 446-451. 